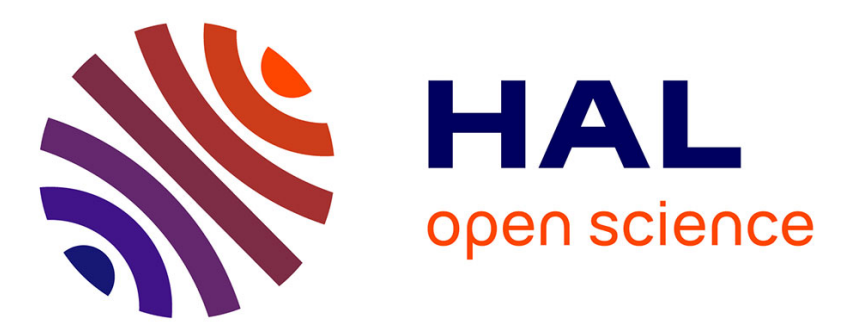

\title{
Spectroscopy of $70 \mathrm{Kr}$ and isospin symmetry in the $\mathrm{T}=$ $1 \mathrm{f}$ pg shell nuclei
}

M M Debenham, M M Bentley, J J Davies, H Haylett, G G Jenkins, P. Joshi, L F Sinclair, R Wadsworth, P Ruotsalainen, J. Henderson, et al.

\section{- To cite this version:}

M M Debenham, M M Bentley, J J Davies, H Haylett, G G Jenkins, et al.. Spectroscopy of 70Kr and isospin symmetry in the $\mathrm{T}=1 \mathrm{f}$ pg shell nuclei. Physical Review C, 2016, 94, pp.54311. 10.1103/PhysRevC.94.054311. cea-01481486

\section{HAL Id: cea-01481486 https://hal-cea.archives-ouvertes.fr/cea-01481486}

Submitted on 2 Mar 2017

HAL is a multi-disciplinary open access archive for the deposit and dissemination of scientific research documents, whether they are published or not. The documents may come from teaching and research institutions in France or abroad, or from public or private research centers.
L'archive ouverte pluridisciplinaire HAL, est destinée au dépôt et à la diffusion de documents scientifiques de niveau recherche, publiés ou non, émanant des établissements d'enseignement et de recherche français ou étrangers, des laboratoires publics ou privés. 


\title{
Spectroscopy of ${ }^{70} \mathrm{Kr}$ and isospin symmetry in the $T=1 \mathrm{fpg}$ shell nuclei
}

\author{
D. M. Debenham, ${ }^{*}$ M. A. Bentley, P. J. Davies, T. Haylett, D. G. Jenkins, P. Joshi, L. F. Sinclair, and R. Wadsworth
}

Department of Physics, University of York, Heslington, York, YO10 5DD, United Kingdom

\section{P. Ruotsalainen}

University of Jyvaskyla, Department of Physics, P.O. Box 35, FI-40014, University of Jyvaskyla, Finland and TRIUMF, 4004 Westbrook Mall, Vancouver, British Columbia, Canada V6T 2A3

\author{
J. Henderson \\ Department of Physics, University of York, Heslington, York, YO10 5DD, United Kingdom \\ and TRIUMF, 4004 Westbrook Mall, Vancouver, British Columbia, Canada V6T 2A3 \\ K. Kaneko \\ Department of Physics, Kyushu Sangyo University, Fukuoka 813-8503, Japan
}

K. Auranen, H. Badran, T. Grahn, P. Greenlees, A. Herzaáň, U. Jakobsson, J. Konki, R. Julin, S. Juutinen, M. Leino, J. Sorri, J. Pakarinen, P. Papadakis, P. Peura, J. Partanen, P. Rahkila, M. Sandzelius, J. Sarén, C. Scholey, S. Stolze, and J. Uusitalo University of Jyvaskyla, Department of Physics, P.O. Box 35, FI-40014, University of Jyvaskyla, Finland

H. M. David ${ }^{\dagger}$

Physics Division, Argonne National Laboratory, Argonne, Illinois 60439, USA

G. de Angelis

INFN-Laboratori Nazionali di Legnaro, Viale dell'Universitá 2, 35020 Legnaro, Padova, Italy

W. Korten

CEA Saclay, IRFU, SPHN, F-91191 Gif-sur-Yvette, France

G. Lotay

Department of Physics, University of Surrey, Guildford GU2 7XH, United Kingdom

M. Mallaburn

School of Physics and Astronomy, University of Manchester, Manchester, United Kingdom

E. Sahin

Department of Physics, University of Oslo, N-0316 Oslo, Norway

(Received 21 September 2016; published 14 November 2016)

\begin{abstract}
The recoil- $\beta$ tagging technique has been used in conjunction with the ${ }^{40} \mathrm{Ca}\left({ }^{32} \mathrm{~S}, 2 n\right)$ reaction at a beam energy of $88 \mathrm{MeV}$ to identify transitions associated with the decay of the $2^{+}$and, tentatively, $4^{+}$states in the nucleus

${ }^{70} \mathrm{Kr}$. These data are used, along with previously published data, to examine the triplet energy differences (TED) for the mass 70 isobars. The experimental TED values are compared with shell model calculations, performed with the JUN45 interaction in the $f p g$ model space, that include a $J=0$ isospin nonconserving (INC) interaction with an isotensor strength of $100 \mathrm{keV}$. The agreement is found to be very good up to spin 4 and supports the expectation for analog states that all three nuclei have the same oblate shape at low-spin. The $A=70$ results are compared with the experimental and shell model predicted TED and mirror energy differences (MED) for the mass 66 and 74 systems. The comparisons clearly demonstrate the importance of the isotensor INC interaction in replicating the TED data in this region. Issues related to the observed MED values and their interpretation within the shell model are discussed.
\end{abstract}

DOI: 10.1103/PhysRevC.94.054311

\section{INTRODUCTION}

Isospin symmetry studies in $N \approx Z$ nuclei forms a key

*dmd512@york.ac.uk

${ }^{\dagger}$ Present address: GSI Helmholtzzentrum für Schwerionenforschung GmBH, D-64291 Darmstadt, Germany. research area in nuclear structure physics. For example, studies of charge-symmetry breaking terms of the nuclear interaction are vital for the modeling of superallowed Fermi decays 
[1,2]; which is a fundamental topic in nuclear physics for the application of the isospin concept. In a further example, shell-model calculations, intended to predict the location of the driplines, have recently had isospin nonconserving (INC) interactions specifically included [3]. To date, such studies have largely focused on nuclei in the $s d$ and lower $f p$ shells and has involved studies of the energies of analog states (see $[4,5]$ and references therein) as well as studies of the hinderance of $E 1$ decays due to selection rules in $N=Z$ nuclei [6-8]. Detailed investigations in the $f_{\frac{7}{2}}$ shell region have revealed a need to include an isospin nonconserving (INC) force beyond the Coulomb interaction (see Ref. [4]) in order to understand the excited states of isobaric multiplets across the $N=Z$ line in this region. More recent work on data from the lower $f p$ shell region has also shown that the matrix elements associated with the INC term have a strong dependence with the coupled angular momentum, $J$, of the valence particles [1]. At this time, however, the exact origin of the INC term remains unclear. Furthermore, there are still questions as to whether, and how, the term evolves for different shells.

An important feature of the above studies is the assumption that the analog nuclei have identical shapes. Over the last few years developments in experimental techniques have provided the possibility to study analog states belonging to nuclei in the heavier, $N \approx Z$, (upper $f p$ shell) mass $60-80$, region. A key issue with nuclei residing in the middle of this region is the presence of co-existing nuclear shapes at relatively low excitation energies, which result from large shell gaps that are predicted to exist for both oblate and prolate shapes for nuclei with neutron and proton numbers of 34, 36, 38 (see [9,10]). For mass 70 nuclei, in particular, the difference in energy between the prolate and oblate minima in the potential energy surface is sufficiently small that Coulomb effects on the valence particles could result in changes in the degree of mixing between the oblate and prolate wave function components for different excited states. This in turn could lead to different mean-field shapes for the $T=1$ states in the analog nuclei and, as a consequence, the breaking of isospin symmetry [11].

For the analog pair of nuclei ${ }^{70} \mathrm{Se}-{ }^{70} \mathrm{Br}$ an unexpected, negative, trend was found for the Coulomb energy differences (CED) between members of the $T=1$ states as a function of angular momentum compared with the observations for other analog systems in the region, see Fig. 13 in Ref. [12]. The normal, positive, trend in the behavior of the CED as a function of spin has previously been attributed to Coriolis antipairing effects [13]. On the other hand, the negative trend observed for the mass 70 pair was initially attributed to the Thomas-Erhman shift [14], but a different interpretation, based on a prolate shape evolution of the nuclei with increasing spin, has also been suggested [15]. Other, theoretically based, interpretations regarding the anomalous behavior of the mass 70 systems have been proposed. One involves the polarization effects of the valence particles through the isospin-breaking Coulomb interaction. The resulting modification induced on the proton single-particle energies causes changes in the mixing of competing shapes with strong effects on the CED and on the shape which is most stable [11]. These calculations were performed using the excited VAMPIR approach (which allows different shape mixing as a function of spin) and they suggest that the low-spin mean shapes for ${ }^{70} \mathrm{Br}$ and ${ }^{70} \mathrm{Se}$ are prolate and oblate, respectively. A second theoretical interpretation, based on the nuclear shell model approach [16], predicts that both

${ }^{70} \mathrm{Br}$ and ${ }^{70} \mathrm{Se}$ have the same oblate shape and suggests that the observed negative CED between the pair can be attributed to neutron excitations from the $f p$ to the $g_{\frac{9}{2}}$ intruder orbit, which is reflected in a sudden enhancement in the electromagnetic spin-orbit term.

The possibility of rapidly changing shapes in the mass 70 region makes the study of Coulomb (CED) and mirror energy differences (MED - the excitation energy differences between analog states in mirror nuclei) potentially difficult to interpret because these are sensitive to monopole effects (single-particle Coulomb shifts), the electromagnetic spin-orbit interaction and changes in nuclear radius or shape as a function of spin. On the other hand triplet energy differences (TED) defined as

$$
\mathrm{TED}_{J, T}=E_{J, T, T_{z}=-1}+E_{J, T, T_{z}=1}-2 E_{J, T, T_{z}=0}
$$

probe different aspects of the two-body interaction. These reflect the difference between the average of the protonproton and neutron-neutron interactions, and the neutronproton interaction. As such, TED are not strongly influenced by the same contributions that affect MED, but they are sensitive to isotensor (multipole) interactions [4], which arise as a result of a Coulomb interaction and/or a nuclear isospin nonconserving (INC) interaction. This makes TED a potentially sensitive probe of contributions from these two terms. Recent experimental and theoretical work on $T_{z}=-1$ nuclei in the mass 70 region [17-19] provides evidence that a $J=0$ INC interaction, with an isotensor strength similar to that required in the $f_{\frac{7}{2}}$ shell, is also needed in the $f p g$ shell region. The important missing data in the region are for states in ${ }^{70} \mathrm{Kr}$; a nucleus in a region where shape mixing is expected to be at its maximum. Here calculations of TED using the shell model and the latest beyond mean-field complex excited VAMPIR approach predict different outcomes for the $A=70$ nuclei. The shell model predicts negative TED values for the $A=66,70,74,78$ analog triplet systems [19], while in the excited Vampir calculations different prolate-oblate mixtures of the wave functions of the yrast states in the $A=70 \mathrm{Kr}, \mathrm{Br}$, Se nuclei result in a small positive TED up to spin 4 and a negative TED for the $A=74 \mathrm{Sr}, \mathrm{Rb}, \mathrm{Kr}$ systems [20].

The present work has used the established [21,22] recoil- $\beta$ tagging (RBT) technique to identify gamma rays in ${ }^{70} \mathrm{Kr}$. This technique has been successfully employed to study several other nuclei in the region, see $[12,15,17,18,23]$.

\section{EXPERIMENTAL DETAILS}

The K130 cyclotron at the Department of Physics of the University of Jyväskylä (JYFL) was used to produce an $88 \mathrm{MeV}^{32} \mathrm{~S}$ beam with a typical beam current ranging between 2-7 pnA. This beam was used to bombard a ${ }^{\text {nat }} \mathrm{Ca}$ target of thickness $800 \mu \mathrm{g} / \mathrm{cm}^{2}$ and backed with a ${ }^{\text {nat }} \mathrm{C}$ charge reset foil, of thickness $50 \mu \mathrm{g} / \mathrm{cm}^{2}$ to produce the nucleus of interest via the ${ }^{40} \mathrm{Ca}\left({ }^{32} \mathrm{~S}, 2 n\right){ }^{70} \mathrm{Kr}$ reaction. Prompt $\gamma$ rays from the fusion evaporation reactions occurring at the target position were detected using the JUROGAM II array which consisted 
of 24 Eurogam II clover detectors [24] and 15 Eurogam phase I/GASP [25,26] type detectors and had an efficiency of $\approx 5.5 \%$ at $1.3 \mathrm{MeV}$. The fusion evaporation recoils, which entered the RITU gas filled separator [27], were transported to the focal plane where they were implanted into a double sided silicon strip detector (DSSSD), which formed part of the Gamma Recoil Electron Alpha Tagging (GREAT) [28] spectrometer. Located upstream of the GREAT detector system was a multiwire proportional counter (MWPC), which was used to register the recoils being implanted into the DSSSD. This detector generates a $\Delta E$ signal for the recoils, with the remainder of the recoil energy being deposited in the DSSSD. The DSSSD consisted of $120 x$ and $80 y$ strips, each of width $\approx 0.5 \mathrm{~mm}$, and $500 \mu \mathrm{m}$ thick. The gains were set such that the $x$ strips recorded events up to $\sim 12 \mathrm{MeV}$ and the $y$ strips up to $\approx 1 \mathrm{MeV}$, and the thresholds were set to ensure that a $\beta$ signal could be obtained above the noise. Immediately behind this DSSSD was the GREAT [28] planar germanium detector. This allowed the detection of low energy $\gamma$ rays at the focal plane and also, when used in combination with the DSSSD, acted as a $\Delta E-E$ telescope for the $\beta$ particles. Time of flight (ToF) information was generated from events detected in the MWPC and the DSSSD. Data were recorded with the triggerless total data readout (TDR) [29] acquisition system for $235 \mathrm{~h}$ and analysed using the GRAIN software [30]. Based on an average 4 pnA beam the estimated cross section for the production of ${ }^{70} \mathrm{Kr}$ is of the order of 150-200 nb.

Surrounding the target, but located within the target chamber, was the UoYTube (University of York tube). This was used in order to veto recoil events that come in coincidence with charged particles emitted from fusion evaporation reactions at the target position. The UoYTube consisted of $96 \mathrm{CsI}(\mathrm{Tl})$ crystals in a hexagonal configuration with two end caps [22]. $\mathrm{Ni}$ foils of thickness $10 \mu \mathrm{m}$ thick on the hexagonal panels and $20 \mu \mathrm{m}$ thick on the forward end cap, respectively, were used to stop the scattered beam and carbon/oxygen contaminant recoils from the target/charge reset foil from entering the CsI detectors.

\section{DATA ANALYSIS AND RESULTS}

The known fast $\beta$-decay lifetime of ${ }^{70} \mathrm{Kr}$ [31], its large negative $Q$ value $(>10 \mathrm{MeV})$ and its Fermi-superallowed nature enables the recoil- $\beta$ tagging method to be used in this analysis. This method has the advantage of being able to achieve higher sensitivity compared to fusion evaporation reaction techniques involving the detection of two neutrons and application of a charged particle veto detector for removal of events associated with charged particle emission reaction channels. In the latter case the cross-section limit for $\gamma$ identification is typically around $1 \mu \mathrm{b}$ and hence the reaction in this study is out of reach.

In the present work the reaction channel of interest involves the emission of just two neutrons, while other reaction products involve the emission of at least one charged particle. A tried and tested approach was taken to identify transitions of interest. A timing condition for the correlation time between recoil implantation and the $\beta$ (positron) signal of less than $100 \mathrm{~ms}$ and a high $\beta$ threshold of greater than $2 \mathrm{MeV}$ in the planar Ge detector were used to suppress higher cross section reaction channels, which have longer $\beta$ half-lives and lower end-point energies. As expected, transitions from ${ }^{69}$ As ( $3 p$ channel) and ${ }^{70} \mathrm{Se}(2 p)$ were strong and hence still appeared in the tagged spectrum. To help suppress these, and other charged particle evaporation channels, a requirement was placed on the UoYTube veto detector that no charged particles were in coincidence with the detected recoils. In the present experiment this detector had an efficiency of $73 \%$ for the detection of one proton and a $3 p$ channel veto efficiency of $\approx 99 \%$.

When creating the final spectra a lower software threshold of $60 \mathrm{keV}$ was applied to the DSSSD energy signals. Inspection of the DSSSD-planar coincidence timing revealed the presence of three distinct time distributions. While all components of these distributions contribute to the $\beta$-decay events, one of them was found to be dominated by events from nuclei such as ${ }^{69}$ As ( $3 p$ evaporation channel), that have lower $Q_{\beta}$ values. The other components of the time spectrum were found to have strong correlations with the full energy signal of medium/higher energy $\beta$ decays such as those observed from ${ }^{70} \mathrm{Br}$. Furthermore, DSSSD-planar coincidence timing events that are associated with known $\gamma$ rays in ${ }^{70} \mathrm{Br}$ and the $\gamma$ rays that were initially identified as belonging to the zero charged particle emission channel (see below), ${ }^{70} \mathrm{Kr}$, were found to show similar distributions, as expected for nuclei that have almost the same high $Q_{\beta}$ values. (The highest energy positrons from the ${ }^{70} \mathrm{Br}$ decays give the largest DSSSD-planar time differences.) The above findings allowed a DSSSD-planar time window to be applied in order to select events associated with medium energy $\beta$ decays. This had the effect of providing a small reduction in ${ }^{70} \mathrm{Br} \gamma$ ray contamination in the spectrum used to identify the ${ }^{70} \mathrm{Kr}$ transitions (see below).

Figure 1(a) shows $\gamma$-ray events that correlate with a highenergy positron $(>2 \mathrm{MeV})$ in the DSSSD-planar telescope within $400 \mathrm{~ms}$ of the correlated recoil implantation, and in addition, that there were no charged particles detected in the UoYTube. (Note, ${ }^{70} \mathrm{Br}$ events dominate this spectrum due to the fact that the UoYTube can only detect one proton events with an efficiency of $73 \%$ and this nucleus is populated via the $p n$ channel.) The spectrum shown in Fig. 1(b) has the same conditions as in (a) except that the correlation time between the implanted recoil and the $\beta$ particle is now reduced to $100 \mathrm{~ms}$. Figure 1(c) has the same conditions as Fig. 1(b) plus an additional time restriction placed on the DSSSD-planar times (as discussed above) to select only the medium energy $\beta$ particles. The final spectrum shown in Fig. 1(d) has the same conditions as Fig. 1(c) except that in this case there was one charged particle explicitly demanded in the UoYTube. Comparing Figs. 1(a)-1(c) reveals the presence of previously unknown transitions of energies 870(1) keV and very tentatively $997(1) \mathrm{keV}$. The latter transition has four counts and the average background count in the spectrum in the vicinity of this tentative peak is 0.25 counts/channel. The significance of these events is within the $95 \%$ confidence limit, which correspond to statistically significant results in the $2 \sigma$ limit [32]. Both of these $\gamma$ rays are absent in Fig. 1(d), which requires one charged particle to be registered in the UoYTube. The latter spectrum therefore suggests that the 


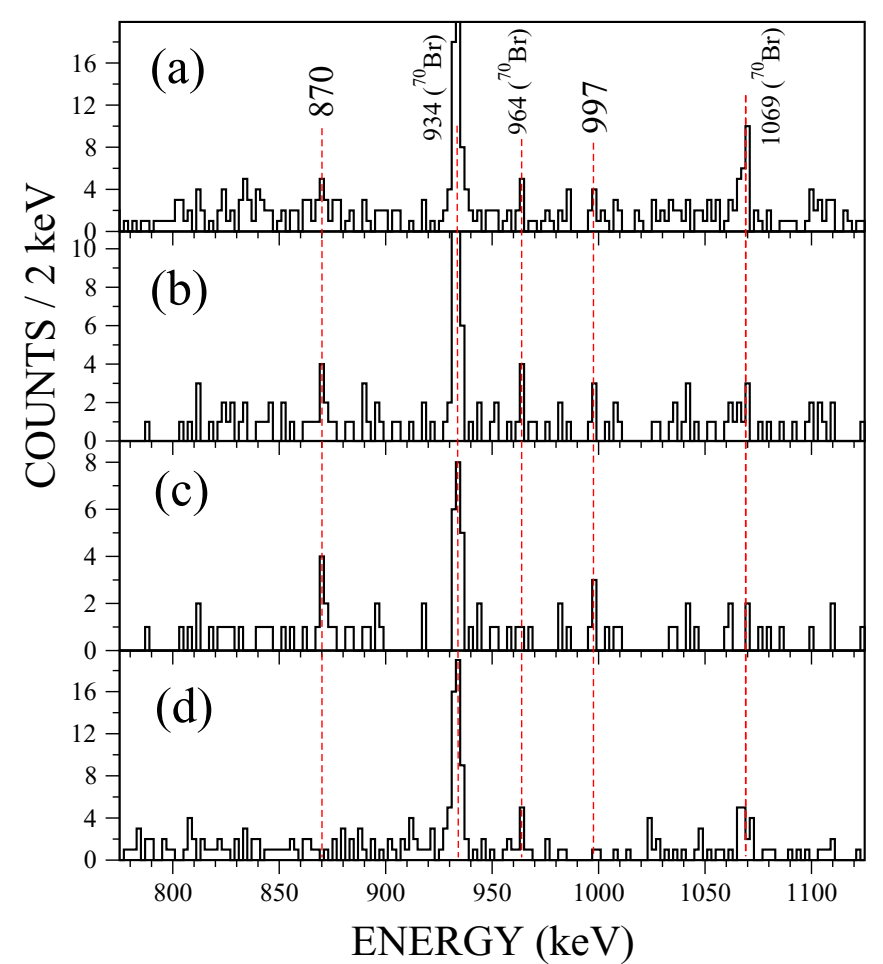

FIG. 1. $\gamma$-ray spectra obtained with JUROGAM II for different tagging conditions. (a) requires that a $\beta$-decay event occurs within $400 \mathrm{~ms}$ of the correlated recoil ion implant, that it has a high-energy positron $(>2 \mathrm{MeV}$ ) recorded in the GREAT planar detector and that no coincident charged particles were recorded in UoYTube; (b) has the same gating conditions as (a) except that the $\beta$-ion correlation time in this case was up to $100 \mathrm{~ms}$, (c) has the same conditions as (b) plus a time restriction on the DSSSD-planar coincidence times to select the medium-energy $\beta$ particles. This reduced the ${ }^{70} \mathrm{Br}$ events in the spectrum-see text for details; (d) same as (c) but with one charged particle explicitly demanded in UoYTube.

two new transitions do not originate from a charged-particle evaporation channel.

From the data it is possible to estimate the half-life associated with the 870 and $997 \mathrm{keV} \gamma$ rays. The spectrum shown in Fig. 2 has gates placed on the above $\gamma$ rays plus additional requirements detailed in the figure caption. The Schmidt method [33] was used, with a single exponential component fit, to extract the mean-lifetime for these data and the method described in [34] was used to determine the relevant asymmetric errors. These results were then converted into half-lives with appropriate errors. The result of this analysis for the sum of the events recorded in the two $\gamma$ rays can be seen in Fig. 2. In this case a half-life of $31_{-7}^{+13} \mathrm{~ms}$ is found. This value is in reasonable agreement with the most recent value reported in the literature for ${ }^{70} \mathrm{Kr}$ of $40 \pm 6 \mathrm{~ms}$ [31]. In order to crosscheck the method, a half-life of $81_{-5}^{+6} \mathrm{~ms}$ was obtained for events associated with the known 321, 403, and $934 \mathrm{keV}$ transitions in ${ }^{70} \mathrm{Br}$ using the same conditions to those specified in Fig. 2, with the exception that in this case one charged particle was required in UoYTube. The result obtained is in good agreement with the currently accepted value of 79.1(8) $\mathrm{ms}$ [35] for the ground-state decay lifetime.

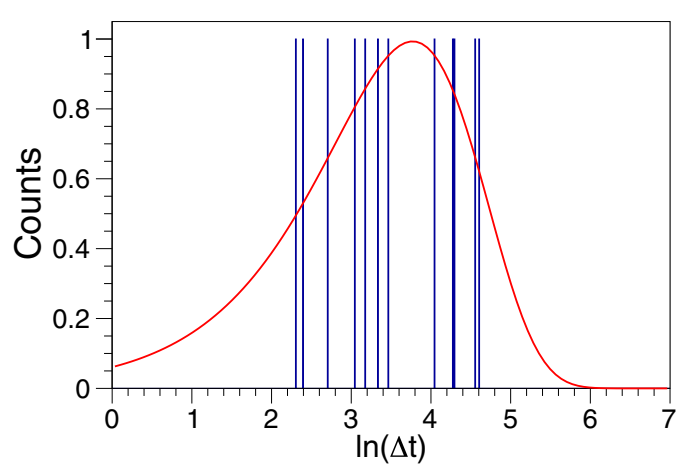

FIG. 2. Natural logarithm, $\ln (\Delta t)$, of decay data gated on the 870 and $997 \mathrm{keV}$ transitions assigned to ${ }^{70} \mathrm{Kr}$, where $\Delta t$ is the time difference in ms between the recoils associated with the two $\gamma$ rays and their subsequent $\beta$ decays. All correlated events within the $500 \mathrm{~ms}$ tagger time range were accepted. In addition to the $\gamma$ ray gates listed above the spectrum shown required a $\beta$-particle energy $>2 \mathrm{MeV}$ in the planar Ge detector and had a time gate on the DSSSD-planar coincidence times to select the medium energy $\beta$ particles. The solid red line represents the Schmidt (log likelihood) method [33] fit to the data. The centroid of the fit yields the mean lifetime $\left(44_{-10}^{+19} \mathrm{~ms}\right)$, which can then be converted to a half-life of $31_{-7}^{+13} \mathrm{~ms}$. See text for comments on the error analysis.

Given that the newly identified transitions are associated with the zero charged particle emission channel and that they have a half-life that is consistent with the most recently reported value for the ${ }^{70} \mathrm{Kr}$ ground-state decay we assign them to this nucleus.

\section{DISCUSSION}

Based on the observed $\gamma$-ray intensities we tentatively assign the $870 \mathrm{keV} \gamma$ ray to the $2^{+} \rightarrow 0^{+}$decay in ${ }^{70} \mathrm{Kr}$. (A parallel investigation at RIKEN using knockout and inelastic excitation reactions to populate excited states in this nucleus supports, within errors, the positioning of the $2^{+}$state [36].) The observed intensity of the $997 \mathrm{keV}$ transition tentatively suggests that this may be the $4^{+} \rightarrow 2^{+}$decay. Assuming the above assignments, it is possible to evaluate the experimental TED values for the $A=70$ triplet. The results are shown in Fig. 3(b). As discussed in Ref. [4], the reason that TED are always found to be negative results from the fact that they are dependent on the isotensor component of the two body interaction $\left(V_{p p}+V_{n n}-2 V_{n p}\right)$. The decrease in values with spin results from (a) the fact that the number of $T=1 \mathrm{np}$ pairs, for a given analog state, is larger in the odd-odd nucleus than for that of the two even-even nuclei and (b) the Coulomb isotensor interaction is positive and reduces relative to the ground state for increasing angular momentum. Figure 3(b) compares the experimental TED values with results of shell model calculations performed with the JUN45 interaction in the $p f_{\frac{5}{2}} g_{\frac{9}{2}}$ model space [19]. The experimental values clearly agree very well with the theoretical predictions, which include a $J=0$ INC isospin nonconserving interaction, with an isotensor strength of $100 \mathrm{keV}$, in addition to the Coulomb, multipole and monopole terms, the latter of which includes electromagnetic spin-orbit effects [16] in this region. This conclusion is in 


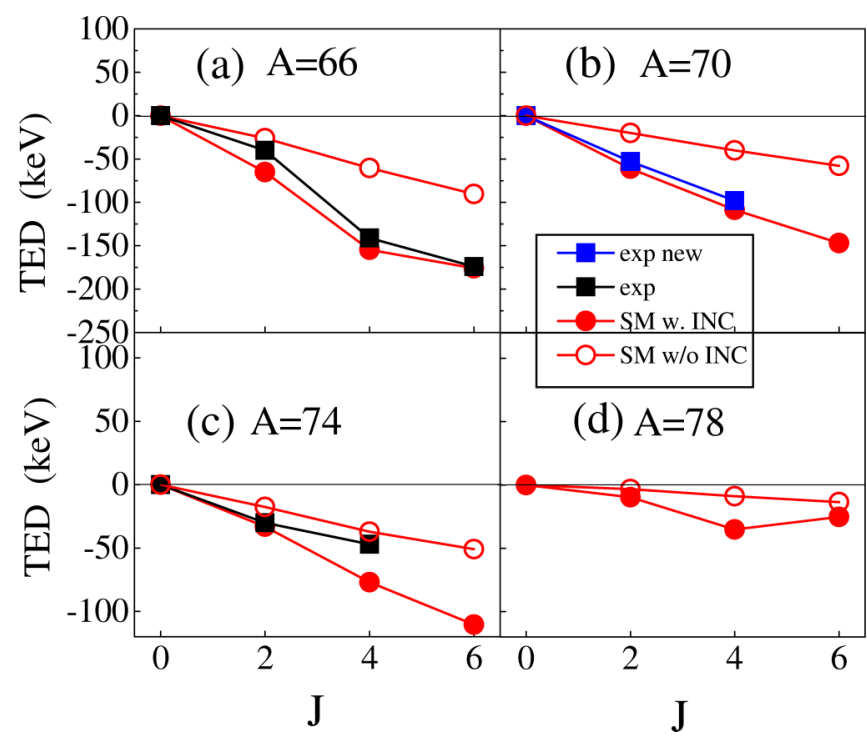

FIG. 3. Triplet energy differences as a function of spin, $J$, for the $A=66,70,74$, and 78 triplets. Black squares show the experimental values for the $A=66$ and 74 systems, blue squares show the new experimental values for the $A=70$ triplet, whilst the solid (open) red circles show the results from shell model calculations with (without) the INC term.

agreement with the conclusions drawn from the $A=66$ and 74 triplet studies [12,18]—see Fig. 3. However, for the $A=74$ triplet there is evidence for the experimental TED departing at spin 4 from the shell model predictions with the INC interaction included [Fig. 3(c)]. A possible, tentative, explanation for this observation was put forward in terms of weak binding effects for the $4^{+}$state [18], but clearly the identification of higher spin states in ${ }^{74} \mathrm{Sr}$ will be important to determine if the level of disagreement persists as the spin increases.

The present results suggest that the beyond mean-field complex excited VAMPIR variational model calculations are not able to reproduce the TED values at this time-see Fig. 6 from [20]. The TED values in these calculations are influenced by the predicted evolution of shape mixing with increasing spin, which is very different for ${ }^{70} \mathrm{Se}$ compared to the other two analog nuclei. It may be that the inclusion of more configurations in VAMPIR will be required in order to reproduce the experimental TED values, which in turn may affect the overall shapes associated with the states in the three nuclei. However, the shape mixing in this model is also known to be sensitive to small changes in the effective Hamiltonian. The present results will hopefully aid further development of the model, which has been reasonably successful in describing other experimental results in the region. From the shell model calculations, the yrast states in ${ }^{70} \mathrm{Kr},{ }^{70} \mathrm{Br}$, and ${ }^{70} \mathrm{Se}$ are all predicted to have positive quadrupole moments (i.e., oblate shapes-see Table I). This is consistent with expectations that analog states correspond to nuclei with the same intrinsic shapes and also with the latest experimental results for ${ }^{70} \mathrm{Se}[37]$.

Mirror energy differences give direct information on the isovector interaction $\left(V_{p p}-V_{n n}\right)$ in nuclei. A detailed analysis, extended to the full $f_{\frac{7}{2}}$ shell, has revealed that it is also necessary to include an INC term for the isovector interaction
TABLE I. Quadrupole moments (in $e \mathrm{fm}^{2}$ ) predicted by the shell model using the JUN45 interaction in the $f p g$ model space (with the INC interaction included) for the yrast states in the $A=70$ triplet nuclei.

\begin{tabular}{llll}
\hline \hline$J^{\pi}$ & ${ }^{70} \mathrm{Se}$ & ${ }^{70} \mathrm{Br}$ & ${ }^{70} \mathrm{Kr}$ \\
\hline $2^{+}$ & 37.3 & 39.8 & 44.4 \\
$4^{+}$ & 49.7 & 54.2 & 59.6 \\
$6^{+}$ & 55.1 & 59.9 & 65.7 \\
\hline \hline
\end{tabular}

to account for the MED (see Refs. [1,4,38]). Indeed, an INC term of $+100 \mathrm{keV}$ for (just) the $J=2$ coupling has been used successfully in order to provide a correct description of the MED throughout the $f_{\frac{7}{2}}$ shell $[4,38]$. This has been shown [1] to be largely equivalent to using $-100 \mathrm{keV}$ for the $J=0$ term instead. A full set of $J$-dependent INC terms has been shown to further improve agreement with the data [1,39]. However in all this analysis, the consistent observation in this region is that the $J=0 \mathrm{INC}$ term is required to be about $100 \mathrm{keV}$ below the $J=2$ term in order to account for the data, i.e., there is a $J$ dependence of a specific sign. There have been, until recently, few data on MED in the upper $f p$ shell, although new data on the $A=66,67$, and 74 mirror nuclei $[17,18,40]$ have yielded information at intermediate spins which might begin to shed light on INC interactions in the upper $f p$ shell. A shell-model analysis has been performed for these mirrored systems $[19,41]$, but up to this point a consistent picture is yet to emerge.

Figure 4 shows the experimental MED data (squares) for the $A=66,70$, and 74 mirror pairs and compares the results with shell model calculations in the $f_{5 / 2} \mathrm{pg}_{9 / 2}$ model space with the JUN45 interaction-see Ref. [19] for details. The shell-model result without the inclusion of any INC parameters

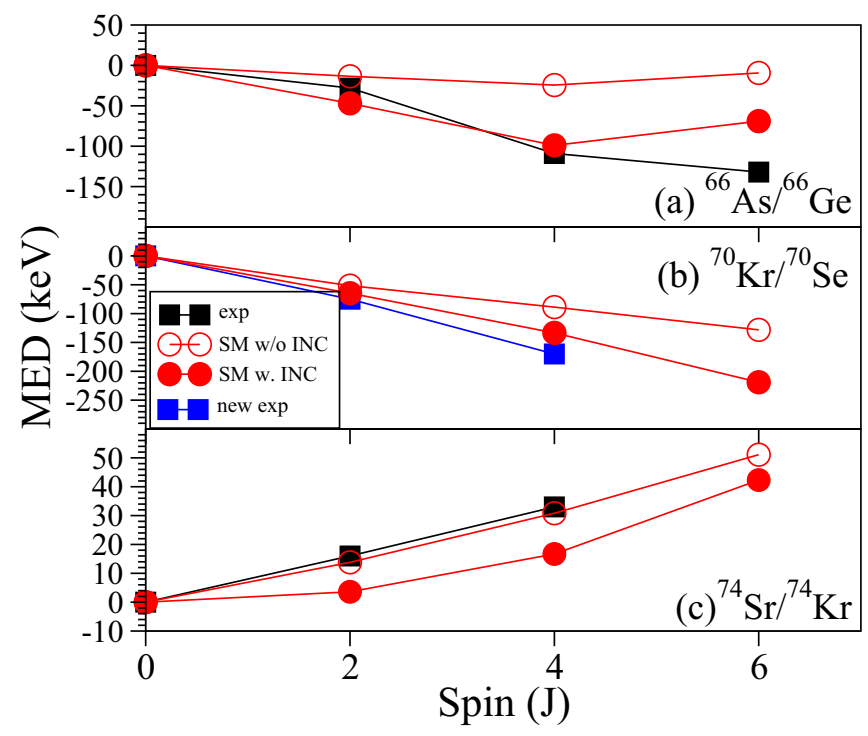

FIG. 4. Mirror energy differences as a function of spin, $J$, for the $A=66,70$, and 74 mirror pairs. Black squares show the experimental values for the $A=66$ and 74 systems, blue squares show the new experimental values for the $A=70$ mirror pair, while the solid (open) red circles show the results from shell model calculations with (without) the INC interaction included. 
is shown (empty circles) and, while $A=74$ is reproduced well, the agreement is poor for higher-spin states in $A=66$ and (now) $A=70$. When implementing INC interactions in these calculations, it was suggested in Ref. [19] that an improved agreement for the higher-spin $A=66 \mathrm{MED}$ is obtained when an INC value of $+300 \mathrm{keV}$ at $J=0$ is used. The shell-model results with this INC term included are shown (filled circles) for all three pairs. The agreement for $A=66$ and 70 is much improved, although $A=74$ is slightly worsened. Given that the effective INC terms may well have a nuclear structure origin (see, e.g., [1]), it is not known how consistent they should be between different mass regions. However, it is worth noting that an INC term of $+300 \mathrm{keV}$ at $J=0$ is very different from the observation in the lower $f p$ shell, having both a different magnitude and sign of the $J$ dependence. One issue that is expected to complicate the ability to replicate experimental MED in this region is the more complex valence space in the upper- $f p$ shell compared with the $f_{\frac{7}{2}}$ shell, where one orbital dominates the wave functions. In this $A=70$ region, it is already seen [19] that the single-particle (Coulomb and spin-orbit) contributions to the MED are large, especially at higher spins, and these will depend critically on the detailed structure of the wave functions predicted by the shell model. Furthermore, it is highly likely that a more extended model space, such as fpgd may be required by shell model calculations, especially to describe the MED given that the detailed configurations may affect the single-particle MED terms. It is also clear that further MED data will be required to tie down the effective isovector INC in this region.

\section{SUMMARY}

Using the recoil- $\beta$ tagging technique the present work has enabled the tentative identification of two $\gamma$ rays in ${ }^{70} \mathrm{Kr}$. Under the assumption that these represent the yrast $2^{+} \rightarrow 0^{+}$ and $4^{+} \rightarrow 2^{+}$transitions this has allowed a comparison to be carried out with recently published shell model calculations [19] for triplet energy differences with and without the $J=0$ isospin nonconserving (INC) interaction included. As in the case of the $A=66$ system the level of agreement for the calculations with the INC isotensor interaction of strength $100 \mathrm{keV}$ included is very good. The new results for ${ }^{70} \mathrm{Kr}$ also suggest that earlier predictions [20] of different shapes for the low-lying $T=1$ states across the isospin triplet appears not to be valid. It is clear that there is an urgent need for knowledge of higher-spin $T=1$ states in the even-even $A \geqslant 70$ nuclei. Furthermore, the apparent success of the shell model calculations at low-spin needs testing further through the measurement of electromagnetic transitions strengths. However, in order to replicate these it is expected that it may be necessary to move to an $f p g_{\frac{9}{2}} d_{\frac{5}{2}}$ model space, which is an on-going challenge for the shell model [42]. The experimental results for the MED in this mass region clearly indicate that an isovector INC interaction is needed within the shell model calculations, however, further experimental and theoretical work are required to understand the interaction strength required.

\section{ACKNOWLEDGMENTS}

This work has been supported by the Academy of Finland under the Finnish Center of Excellence Programme (20122017). The authors also thank the GAMMAPOOL European Spectroscopy Resource for the loan of the detectors for the JUROGAM II array. Support has also been provided by the EU 7th framework programme, Project No. 262010 (ENSAR). We wish to acknowledge support from the UK STFC under Grants No. ST/J000124 and No. ST/L005727. J.H. and P.R. acknowledge the support of the Natural Sciences and Engineering Research Council of Canada.
[1] M. A. Bentley, S. M. Lenzi, S. A. Simpson, and C. Aa. Diget, Phys. Rev. C 92, 024310 (2015).

[2] I. S. Towner and J. C. Hardy, Phys. Rev. C 77, 025501 (2008).

[3] K. Kaneko, Y. Sun, T. Mizusaki, and S. Tazaki, Phys. Rev. Lett. 110, 172505 (2013).

[4] M. A. Bentley and S. M. Lenzi, Prog. Part. Nucl. Phys. 59, 497 (2007).

[5] Y. H. Lam, N. A. Smirnova, and E. Caurier, Phys. Rev. C 87, 054304 (2013).

[6] E. Farnea et al., Phys. Lett. B 551, 56 (2003).

[7] A. Corsi et al., Phys. Rev. C 84, 041304(R) (2011).

[8] S. Ceruti et al., Phys. Rev. Lett. 115, 222502 (2015).

[9] W. Nazarewicz et al., Nucl. Phys. A 435, 397 (1985).

[10] M. Bender, P. Bonche, and P. H. Heenen, Phys. Rev. C 74, 024312 (2006).

[11] G. de Angelis et al., Phys. Rev. C 85, 034320 (2012).

[12] P. Ruotsalainen et al., Phys. Rev. C 88, 024320 (2013).

[13] C. D. OLeary et al., Phys. Lett. B 525, 49 (2002).

[14] G. de Angelis et al., Eur. Phys. J. A 12, 51 (2001).

[15] B. S. Nara Singh et al., Phys. Rev. C 75, 061301(R) (2007).
[16] K. Kaneko, T. Mizusaki, Y. Sun, S. Tazaki, and G. deAngelis, Phys. Rev. Lett. 109, 092504 (2012).

[17] P. Ruotsalainen et al., Phys. Rev. C 88, 041308(R) (2013).

[18] J. Henderson et al., Phys. Rev. C 90, 051303(R) (2014).

[19] K. Kaneko, Y. Sun, T. Mizusaki, and S. Tazaki, Phys. Rev. C 89, 031302(R) (2014).

[20] A. Petrovici, Phys. Rev. C 91, 014302 (2015).

[21] A. Steer et al., Nucl. Instrum. Methods Phys. Res. A 565, 630 (2006).

[22] J. Henderson et al., J. Instrum. 8, P04025 (2013).

[23] H. M. David et al., Phys. Lett. B 726, 665 (2013).

[24] G. Duchêne et al., Nucl. Instrum. Methods Phys. Res. A 432, 90 (1999).

[25] C. W. Beausang, Nucl. Instrum. Methods Phys. Res. A 313, 37 (1992).

[26] C. Rossi Alvarez, Nucl. Phys. News 3, 10 (1993).

[27] M. Leino et al., Nucl. Instrum. Methods Phys. Res. B 99, 653 (1995).

[28] R. D. Page et al., Nucl. Instrum. Methods Phys. Res. B 204, 634 (2003). 
[29] I. Lazarus et al., IEEE Trans. Nucl. Sci. 48, 567 (2001).

[30] P. Rahkila, Nucl. Instrum. Methods 595, 637 (2008).

[31] A. M. Rodgers et al., Nucl. Data Sheets 120, 41 (2014).

[32] G. J. Feldman and R. D. Cousins, Phys. Rev. D 57, 3873 (1998).

[33] K.-H. Schmidt et al., Z. Phys. A 316, 19 (1984).

[34] R. Barlow, Nucl. Instrum. Meth. Phys. Res. A 550, 392 (2005).

[35] J. K. Tuli, Nucl. Data Sheets 103, 389 (2004).

[36] K. Wimmer, T. Arici et al. (unpublished).
[37] J. Ljungvall et al., Phys. Rev. Lett. 100, 102502 (2008).

[38] A. P. Zuker, S. M. Lenzi, G. Martínez-Pinedo, and A. Poves, Phys. Rev. Lett. 89, 142502 (2002).

[39] S. A. Milne et al., Phys. Rev. C 93, 024318 (2016).

[40] R. Orlandi et al., Phys. Rev. Lett. 103, 052501 (2009).

[41] K. Kaneko, S. Tazaki, T. Mizusaki, Y. Sun, M. Hasegawa, and G. deAngelis, Phys. Rev. C 82, 061301(R) (2010).

[42] K. Kaneko, T. Mizusaki, Y. Sun, and S. Tazaki, Phys. Rev. C 89, 011302(R) (2014); 92, 044331 (2015). 